verified in vitro, and its targeting pathways were also investigated.

Results $\mathrm{sEV}$ s derived from radiated cells exhibited a stronger MDSCs induction capacity and MDSCs were more suppressive of $\mathrm{T}$ cell proliferation. MDSCs in ESCC bearing mice after radiation increased and the suppressive effect of MDSCs was improved. RNA-seq showed up-regulated PI3K-AKT pathway activity in MDSCs in ESCC bearing mice after radiation. sEVs from the plasma of ESCC-bearing mice after radiation-induced more MDSCs and had a stronger ability to induce immunosuppressive function of MDSCs. miRNA-26b in sEVs of ESCC cell lines and plasma of tumour-bearing mice were found to be up-regulated after radiation. miRNA-26b was found to have the ability to induce MDSCs expansion and function. miRNA-26b was found to activate PI3K-AKT pathway through targeting Pten.

Conclusions sEVs from ESCC cells after ionizing radiation had a stronger ability to induce MDSCs than sEVs 0gy in both promoting cell expansion and immunosuppressive function. In vivo study validated the MDSC-inducing function of radiation.

\section{IDDF2021-ABS-0169 THE ASSOCIATION BETWEEN EXERCISE, NUTRIENT AND FOOD HABITS WITH THE SINGAPOREAN GUT MICROBIAL COMPOSITION}

${ }^{1}$ Shiang-Chiet Tan* ${ }^{1}$ Kai-Yee Toh, ${ }^{1}$ Jia-Pei Ho, ${ }^{2}$ Chun-Wie Chong, 'Jeremy Fung-Yen Lim ${ }^{3}$ Jonathan Wei-Jie Lee. ${ }^{1}$ Asian Microbiome Library (AMILI), Singapore; ${ }^{2}$ School of Pharmacy, Monash University Malaysia, Malaysia; ${ }^{3}$ Division of Gastroenterology and Hepatology, National University Hospital, Singapore

\subsection{6/gutjnl-2021-IDDF.3}

Background The impact of non-dietary lifestyle factors on the gut microbiota, such as physical activity, has not been frequently reported. Recent studies have suggested that exercise can increase the number of beneficial microbial species which enhance short-chain fatty acid synthesis and carbohydrate metabolism. Therefore, we investigated the relationship between physical activity and gut microbiome composition within a cohort of multi-ethnic healthy Singaporeans.

Methods 257 individuals completed the Global Physical Activity Questionnaire (GPAQ) (Armstrong et al. 2006) and Food Frequency Questionnaire (FFQ) (Whitton et al. 2017), to obtain quantitative measures of their physical activity, nutrition and dietary habits, respectively. They also provided paired stool samples, which underwent 16S rRNA V3-4 amplicon sequencing. Community and individual microbial features associated with physical activity were assessed using microbial ecology measures such as $\alpha$-diversity and principal coordinate analysis (PCA), as well as, multivariate sparse Partial Least Squares analyses, whilst confounding for nutritional and dietary habits.

Results Using the GPAQ, 57\% of the individuals were deemed to have an active lifestyle. Active individuals have increased microbial $\alpha$-diversity, although not statistically significant. Unsupervised PCA analysis identified a subset of active individuals with distinct microbiome signatures, whereby these individuals, compared to those with sedentary lifestyles, have increased abundances of Prevotella spp., which is in keeping with their increased fibre intake. Sedentary lifestyle individuals were found to have increased abundance of Lachnoclostridium spp., whereby phylogenetic similar microbial features (such as Roseburia and Subdoligranulum spp.) were significantly
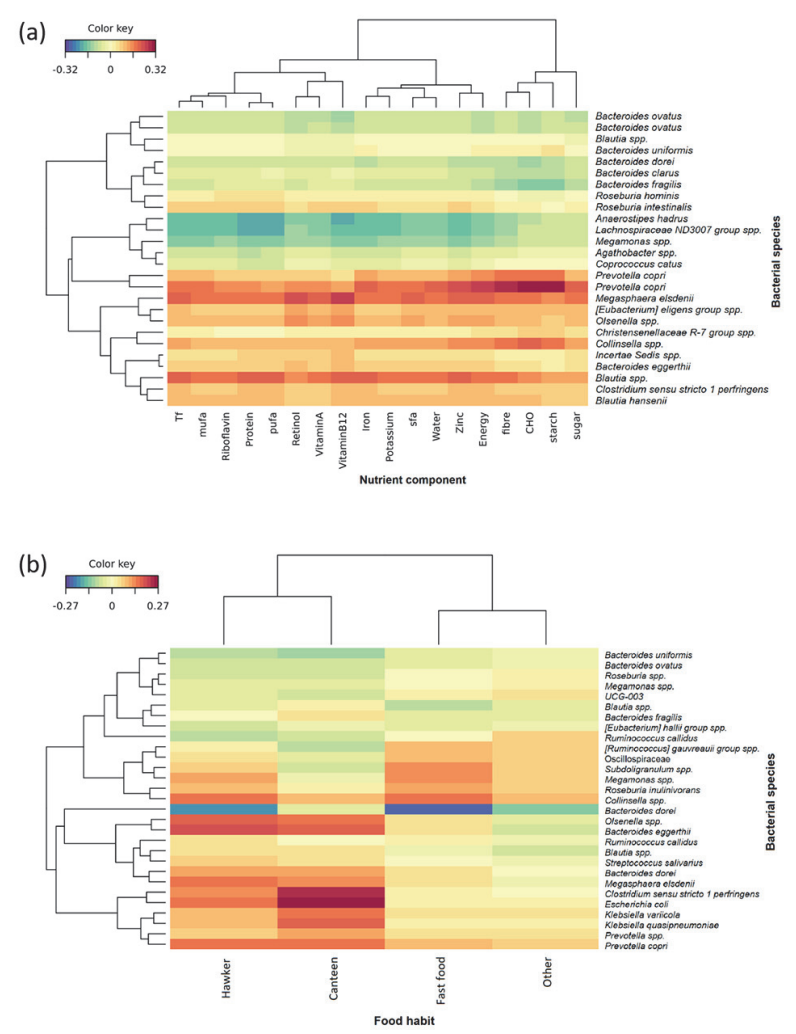

Abstract IDDF2021-ABS-0169 Figure 1

correlated with more frequent eating out at hawker stalls and fast-food establishments (IDDF2021-ABS-0169 Figure 1. Correlation between gut microbiome with (a) nutrient intake; and (b) food habit). Studying the metabolic potential of the microbiome, active individuals had increased abundances of gene content related to energy production, whereby sedentary individuals had increased abundances of gene content related to degradation of aromatic compounds.

Conclusions Our study reaffirms that increased physical activity is associated with increased microbial diversity and beneficial microbial features. However, the differences in gut microbiota associated with physical activity may be due to differences in dietary intake and practices, rather than the exercise itself. Further studies investigating the link of gut microbiome and physical activity should control and standardize the dietary intake of participants.

\section{IDDF2021-ABS-0173 CANCER-ASSOCIATED FIBROBLASTS PROMOTE COLORECTAL CANCER PROGRESSION THROUGH IGF2-IGF1R-YAP1 AXIS}

${ }^{1}$ Bonan Chen*, ' Jinglin Zhang, 'Wai-Nok Chan, ${ }^{2}$ Chi-Chun Wong, ${ }^{2} J u n$ Yu, ${ }^{1}$ Wei Kang, ${ }^{1}$ Ka-Fai To. ${ }^{1}$ Department of Anatomical and Cellular Pathology, State Key Laboratory of Translational Oncology, Li Ka Shing Institute of Health Science, Sir Y.K. Pao Cancer Center, The Chinese University of Hong Kong, Hong Kong; ${ }^{2}$ Institute of Digestive Disease, State Key Laboratory of Digestive Disease, The Chinese University of Hong Kong, Hong Kong

10.1136/gutjnl-2021-IDDF.4 